\title{
Evolución de la Educación Química en nuestro país: una mirada desde la enseñanza y la Didáctica
}

\author{
Mónica Franco ${ }^{1,2}$, Manuel Nieto ${ }^{1,2}$ \\ ${ }^{1}$ Inspección de Química. Consejo de Educación Secundaria. Uruguay \\ ${ }^{2}$ Instituto de Profesores "Artigas". Montevideo - Uruguay \\ monifranco28@gmail.com
}

Recibido13 de octubrede 2020 Aceptado 15 noviembre de 2020

\section{Resumen}

La enseñanza de la Química en el Uruguayfue variando en sus enfoques a lo largo de la historia, siguiendo las tendencias que en general se fueron presentando en educación. Su evolución ha sido similar al camino recorrido por la Biología y la Física, identificándose aspectos comunes en las didácticas de las Ciencias Experimentales, con matices propios. Presentamos en este trabajo un breve análisis de la evolución de la enseñanza de la Química en educación primaria, media y su relación con la formación de los docentes y su didáctica en nuestro país.

Palabras clave: Enseñanza de la Química; Didáctica de la Química

\begin{abstract}
The Chemistry teaching in Uruguay has varied in its approaches throughout history, following the trends that were generally present in education. Its evolution has been similar to the path traveled by Biology and Physics, identifying common aspects in the didactics of Experimental Sciences, with their own nuances. We present in this paper a brief analysis of the evolution of Chemistry teaching in primary and secondary education and its relationship with the training of teachers and their didactics in our country.
\end{abstract}

Key words: chemistry teaching, chemistry didactics. 


\section{Introducción}

Muchos años han transcurrido desde que comenzó en nuestro país la enseñanza de la Química en diferentes niveles. Resulta indiscutible que la evolución que la enseñanza de la Química ha experimentado en el Uruguay, ha seguido las tendencias internacionales, y se ha visto influida -como la enseñanza de otras disciplinas- por las diversas corrientes filosóficas y pedagógicas dominantes en cada época.

Si bien no pretendemos realizar un análisis histórico, es importante ubicar cada etapa de la enseñanza para poder comprender las inclinaciones que se manifiestan en ella.

\section{La educación en la etapa colonial}

En el período colonial se empleó la evangelización como modelo educativo. Únicamente existieron algunas escuelas elementales en las que se enseñaba lo imprescindible para la vida en sociedad, lo que no comprendía ninguna de las ciencias experimentales, de escaso desarrollo por ese entonces. Según Verdesio (1962, pág. 14) “La enseñanza en las escuelas de la Colonia era colectiva y memorística. No había garantía en el contenido y método de enseñanza que se impartía en razón de que los maestros eran improvisados y algunos no tenían preparación ni condiciones pedagógicas".-

\section{La enseñanza de la Química en Educación Primaria}

Durante el transcurso del siglo XX los programas de Educación Primariafueron incluyendo cada vez una mayor cantidad de contenidos de esta disciplina. En los programas vigentes desde el año 2008 se establece que en la enseñanza de las Ciencias Naturales "se emplea un enfoque contextualita que presta una atención especial a la interacción permanente entre la ciencia y la sociedad" (CEIP, 2008, pág.83). En esta área se enseñan saberes provenientes de los diferentes campos disciplinares organizados desde un enfoque multidisciplinar.

Actualmente, la enseñanza de contenidos de Química comienza en Educación Inicial y continúa durante toda la etapa escolar abarcando múltiples contenidos de la asignatura, muchosde los cuales implican importantes niveles de abstracción (por ejemplo modelo corpuscular de la materia: átomos y moléculas). Por ello, resulta difícil pensar en un abordaje mínimamente exhaustivo de los mismos, considerando además que muchos do- centes de primaria poseen escasa formación en contenidos y en Didáctica de la Química.

\section{Los comienzos de la Educación Secundaria}

En los comienzos del Uruguay independiente el Instituto de Instrucción Pública que fuera creado en 1847, tenía a su cargo la Educación Primaria así como la "Secundaria y Científica". A partir de 1849 -año en que se inauguró la Universidad de la República- este Instituto pasó a depender de ella; en ese entonces, los estudios secundarios incluían únicamente Física y Matemática, como disciplinas científicas. Recién en 1854 se sentaron las bases que permitieron crear posteriormente la Educación Secundaria o Preparatoria, la cual contaba con cursos de Química, si bien no se había podido organizar un sistema educativo como tal. Este podría ser considerado como el comienzo de la enseñanza de la Química en el Uruguay, de manera formal.

La reforma impulsada por José Pedro Varela estableció en 1875 un Sistema Educativo Público, en el que se incluyó la Sección Enseñanza Secundaria como parte de una Universidad. En esta época el enfoque positivista con representantes fuertes como Gonzalo Ramirez y José P. Varela se opuso a laintroducción del espiritualismo en la educación, surgiendo una importante polémica. La Sección Secundaria dependiente de la Universidad de la República se mantuvo hasta 1936.A partir de ese momento pasó a constituir un servicio descentralizado del Ministerio de Instrucción Pública, bajo la dirección de un consejo.

\section{Orígenes de la enseñanza técnica}

La enseñanza técnica tiene sus comienzos en nuestro país en el año 1879, al crearse la Escuela de Artes y Oficios, institución destinada originalmente "para menores que recoge la policía por las calles por delitos de robo, vagos y otros que no pudiéndose sujetar, las madres entregan a la policía a fin de que sean corregidos" (Alonzo, 2010); es decir que estaba destinada a niños y jóvenes de "mala conducta" y su función principal consistía en disciplinarlos mediante el aprendizaje de diferentes artes y oficios. A comienzos del 1900 y a impulsos del Dr. Pedro Figari, esta escuela comienza a convertirse en un centro educativo más abierto, formador de mano de obra calificada.

En 1942, en procura de cambiar la imagen de la enseñanza técnica se designa a la institución como "Universidad del Trabajo" (UTU), de modo de estimular a los jóvenes para formarse en determinadas habilidades técnicas manuales, que facilitaran su ingreso al mundo 
del trabajo. No se han encontrado referencias a cursos de Química en ese entonces, ya que la propuesta se dirigía a la formación técnica exclusivamente predominantemente práctica.

\section{La enseñanza de la química a nivel de educación media}

A través de comunicaciones verbales y considerando las menciones de G. Klein en una publicación de su autoría, hemos encontrado referencias a cursos de Química en nuestro país desde 1838 , año en que en la propuesta elaborada por el religioso Dámaso A. Larrañaga los incluye en segundo año de la Enseñanza Secundaria. En años siguientes, se encuentran cursos de esta asignatura en tercero (1884)y/o en cuarto año (1887) del nivel secundario, por entonces como parte de cursos preuniversitarios.

Fue recién en el último período de rectorado universitario de Alfredo Vásquez Acevedo hacia 1900, que se adquirieron materiales para equipar laboratorios -entonces denominados "gabinetes" de Química- venidos de Europa, así como otros de Física, materiales de Geografía y Cosmografía. A estas alturas la formación secundaria era paga al igual que la universitaria y contra ello luchó José Pedro Varela.

Si nos remontamos a los antecedentes más recientes encontramos que en Educación Secundaria se dictaban cursos de Química en tercer y cuarto año en el plan de 1941. En este plan, la enseñanza media consistía en cuatro cursos del primer nivel y dos cursos de características propedéuticas, cuya denominación claramente lo indica: "Preparatorios", en el entendido que en ellos se capacitaba a los alumnos para los estudios universitarios. En todos los programas, se explicitaban los temas a tratar en secuencias de "bolillas" y la bibliografía correspondiente, no encontrándose expresión de los objetivos ni orientación metodológica alguna. Se abordaban primero contenidos de Química General e Inorgánica y luego de Orgánica. En el ciclo superior, en las opciones Medicina, Química e Ingeniería también había cursos de Química. Ellos versaban sobre Química Orgánica, una extensa selección de contenidos de Química General y algo de Inorgánica. Se apelaba fundamentalmente a aprendizajes de tipo memorístico y existían algunos textos cuasi preceptivos en los que se presentaba la secuencia de contenidos de cada curso. Corresponden a esta época textos como los de Química Elemental Moderna de E. Emeric (Uruguay) y de Celsi \& lacobucci (Argentina), tanto para de Orgánica como de Inorgánica en ambos casos, así como otros empleados a modo de "manuales" para los cursos, por ejemplo los de Isidro Más de Ayala y de Otto González Correa (autores uruguayos).

En los cursos de Química de tercero y cuarto raramente se realizaban trabajos experimentales y en ese caso tenían lugar de manera demostrativa. En "Preparatorios", se separaba la parte teórica del "práctico", dividiéndose la clase en subgrupos como se realiza actualmente. Sin embargo, la forma de trabajo era muy diferente: las actividades se distribuían en ciclos de tres tareas experimentales; durante tres semanas el profesor realizaba cada una de las prácticas de manera demostrativa y luego los estudiantes en equipos, volvían a realizar las mismas actividades rotando durante otras tres semanas. En consecuencia, no había nada novedoso al momento de realizar los trabajos por sí mismos, a excepción de la realización de la manipulación correspondiente. Dado que no se permitía que los estudiantes pudieran disponer de la técnica de trabajo durante la realización del práctico, era imprescindible la memorización de la misma. Se realizaban tareas experimentales potencialmente riesgosas que incluían por ejemplo el calentamiento de una mezcla sulfocrómica para obtener de algunos compuestos orgánicos,
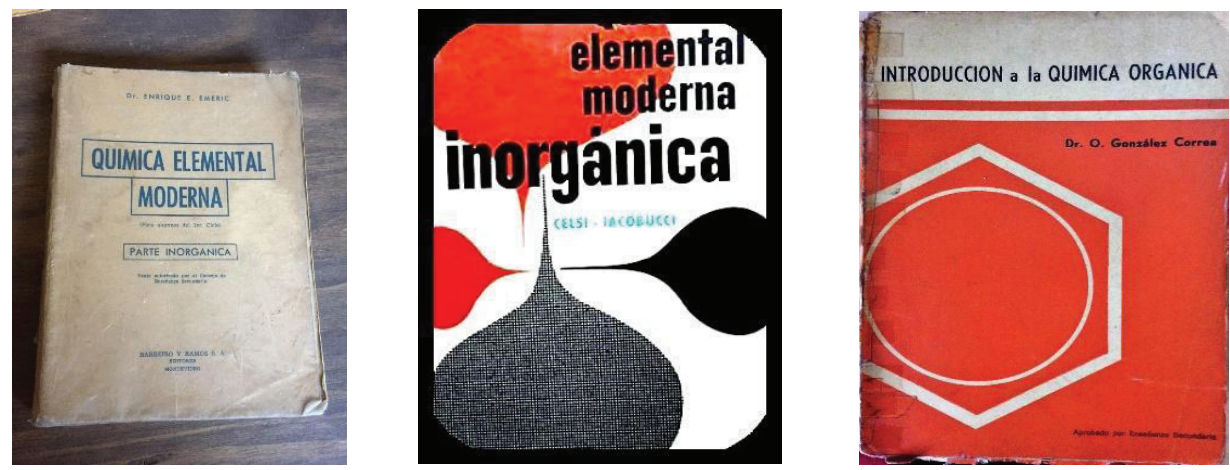

Fig. 1. Algunos libros de texto empleados en los cursos de Química del Plan 1941. 


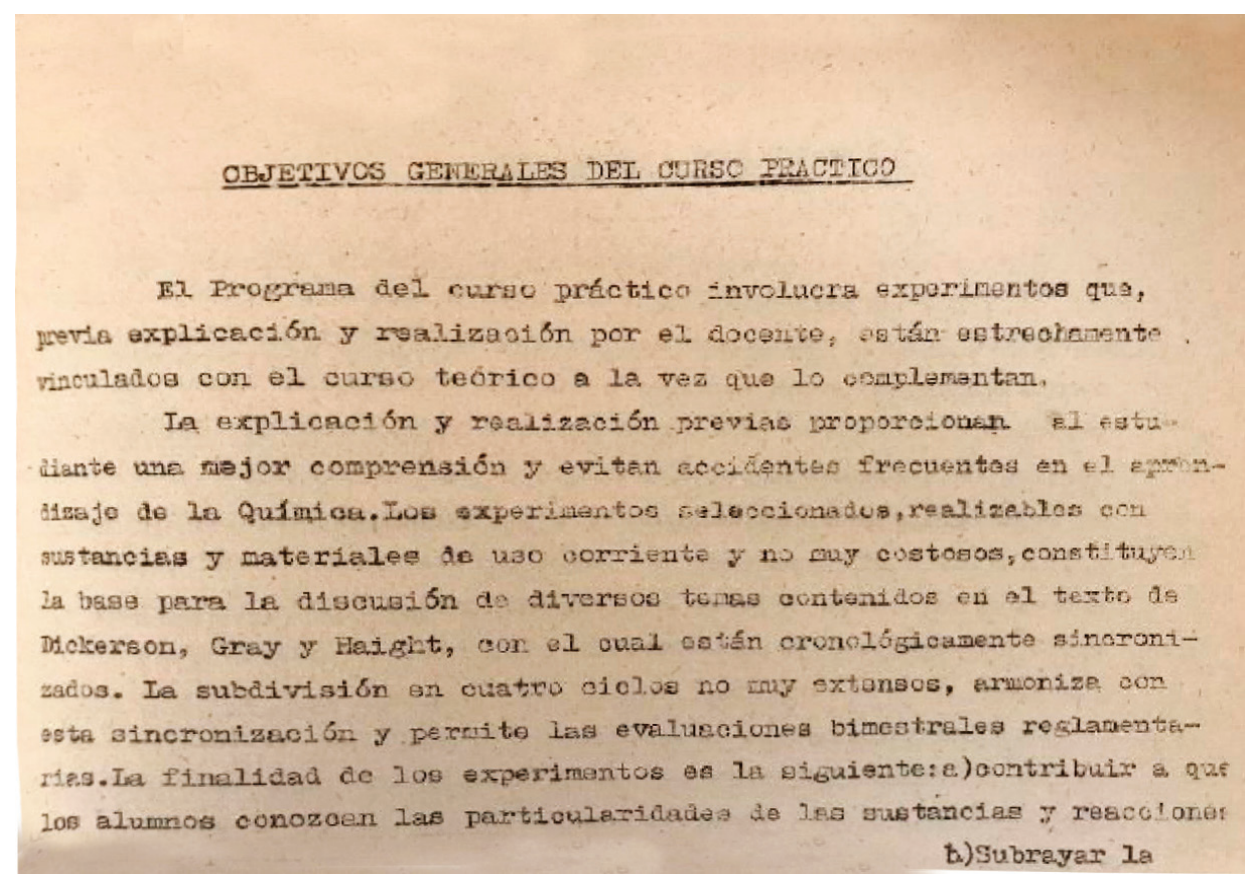

Fig. 2. Extraído del Programa de $2^{\circ}$ año de Preparatorios Medicina Plan 1941.

producción de hidrógeno y cloruro de hidrógeno gaseosos con el riesgo de generar sobrepresión, entre otros peligros.

No debemos olvidar que en el mundo la teoría psicológica conductista emergente en 1913 predominaba en aquel entonces y su arraigo resultaba muy fuerte e influyó en la enseñanza de la Química en varios países del continente por muchos años, incluyendo el Uruguay. En estas etapas, no existía formación para constituirse en docente del sistema educativo a nivel medio.

\section{Plan 63 - Plan 76}

Los cambios sociales de comienzos de los años 60, el inicio de la formación docente específica aproximadamente una década atrás, así como la creciente expansión de la matrícula en Educación Secundaria, provocaron la necesidad de realizar cambios profundos y renovar los planes de estudio.

Las autoridades del momento resolvieron llevar a cabo un proyecto de manera experimental conocido como Plan Piloto, el cual se implementó en algunos liceos de Montevideo y en unos pocos del interior. En este plan los contenidos fueron estructurados en base a núcleos de formación, integrando algunas disciplinas, procurando orientar a los estudiantes según su vocación y aptitudes, en busca de una formación amplia de los jóvenes. No existieron cursos específicos de Química en este plan, sino dos cursos de Ciencias Naturales en el segundo nivel. Este plan no se llegó a extender debido a los altos costos de su implementación, entre otras razones.

A partir del modelo del Plan Piloto, la educación científica comenzó una transformación hacia la enseñanza por descubrimiento, influida por transformaciones educativas de países occidentales del hemisferio norte, donde el desarrollo en ciencia y tecnología eran importantes. Fueron surgiendo entonces proyectos para enseñanza de las ciencias, muchas veces acompañados de capacitación de los docentes por parte de grupos provenientes de esos países. Las propuestas se incluyeron en el Plan 76, el cual introdujo un curso de Química en segundo año del Ciclo Básico (CB). La orientación de los cursos continuó fundándose en la pedagogía del descubrimiento -también llamada de redescubrimiento por quienes sostenían que los aprendices no realizaban verdaderos hallazgos científicos. Esta orientación contó con importante adhesión de los docentes. Los programas de estos cursos de Química contenían indicaciones y objetivos generales con mención a aspectos actitudinales y objetivos conceptuales para cada "capítulo" del programa.

A su vez este plan supuso la separación de la Educación Media en dos ciclos de tres años cada uno: un Ciclo Básico, común a toda ella de carácter obligatorio, y un Bachillerato que incluyó el anterior cuarto año liceal, a 
pesar que el mismo no presentaba las características de los cursos de Bachillerato propiamente, tanto en la metodología de trabajo propuesta como en la forma de evaluación.

Progresivamente se fueron aplicando cambios en el currículo en procura de estimular a los estudiantes, de modo semejante a lo que había ocurrido en EEUU y Gran Bretaña, principalmente. Estos cambios curriculares estuvieron acompañados de algunas capacitaciones para docentes, como una referida al del Chem Study, propuesta que incluía un libro de texto y otro de prácticas para estudiantes, una guía para el docente, - y materiales de apoyo para las clases como por ejemplo películas sobre temáticas del curso. También se emplearon manuales como el Chemical Bonding Approach (CBA) y el curso Introduction to Physical Studies (IPS), cuyo empleo se extendió luego en los cursos de Química de segundo año del Plan.

Estos cursos proponían un cambio de perspectiva de la enseñanza y del papel del docente, que debería consistir mayormente en orientar a los estudiantes en los procesos de descubrir aquellas cuestiones de importancia disciplinar, de manera semejante a cómo lo hacen los científicos. El paradigma del redescubrimiento -ahora extendido a todos los cursos de Química- marcó una diferencia respecto a la enseñanza tradicional que resultaba novedosa. Se planteaba seguir "el método científico", trabajando variadas situaciones experimentales con una secuencia de observación, enunciado de hipótesis, experimentación, análisis de resultados, interpretación y extracción de conclusiones (OHERIC). Sin embargo, los programas de Química del CES en ese entonces incluían solamente un listado de temas a tratar agrupados en unidades, que en algunos casos coincidían con el índice de libro recomendado para el curso.

Las transformaciones iniciadas continuaron teniendo lugar en el período de facto. Las autoridades del Consejo Nacional de Educación (CONAE), consideraron necesario realizar un nuevo cambio de plan. Comenzó una etapa de transición hacia cursos de bachillerato menos propedéuticos, al menos en su denominación. Sin embargo, no se registraron por entonces cambios didácticos ni metodológicos significativos, en general. La forma de trabajo experimental en Química se modificó levemente, dejándose de lado las clases prácticas demostrativas al comienzo de cada ciclo de trabajos experimentales en bachillerato. Sin embargo, en la mayor parte de los casos, las formas de trabajo tradicionales continuaban siendo predominantes, especialmente en el Bachillerato.

En ese período, se crearon los Bachilleratos Tecnológicos en la Universidad del Trabajo, que tenían una extensión un año mayor que los de Secundaria, algunos de los cuales contaban con cursos de Química. En estos cursos los trabajos prácticos se realizaban con la totalidad del grupo, por lo que era difícil que todos los estudiantes tuvieran oportunidad de manipular los materiales de laboratorio, así como de participar activamente de las tareas experimentales.

Con el retorno a la democracia, tuvieron lugar nuevos cambios educativos. El Plan 86 se caracterizó por la búsqueda de acciones compensatorias en el $\mathrm{CB}$, dirigidas a aquellos estudiantes que no lograban niveles mínimos de suficiencia como los cursos de Compensación, durante el año lectivo, y los de Recuperación, a fin de año. Se incorporó en primer y segundo año de CB la asignatura Introducción a las Ciencias Físicas. En primero de Bachillerato (cuarto año) los cursos de Química tuvieron diversificación (Científica y Humanística) con diferencias en los programas y en las cargas horarias (4 horas semanales en orientación Científica y 2 horas en orientación Humanística).

El mayor protagonismo que los estudiantes fueron teniendo en los primeros cursos del CB -especialmente en las tareas experimentales- no se continuaba en los cursos de Química de tercer año, y eran prácticamente
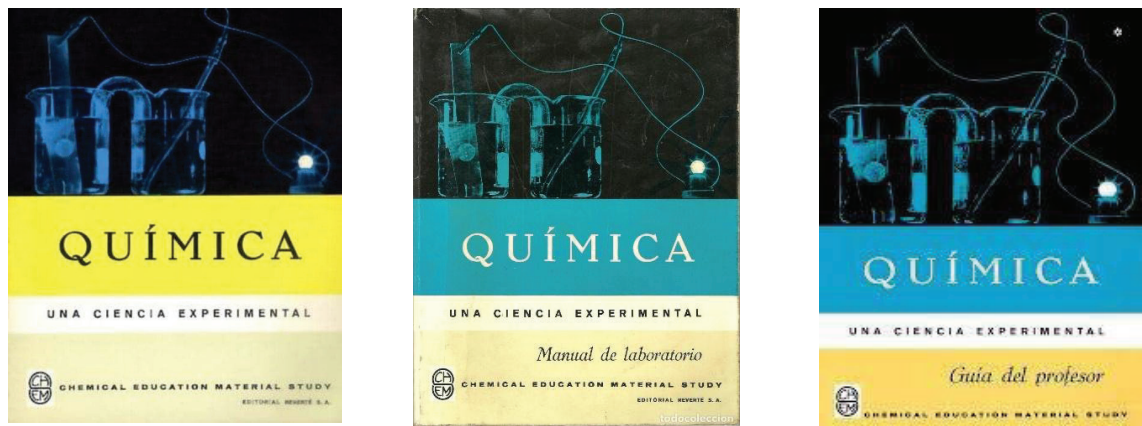

Fig. 3. Química. Una ciencia experimental del programa CHEM Study: Libro del alumno, Manual de Laboratorio y Guía del Profesor. 
inexistentes en los de Primer año de Bachillerato. El abordaje disciplinar en el bachillerato continuó siendo bastante tradicional.

Los programas comenzaron a incluir objetivos generales donde se hace referencia a las actitudes que se pretende desarrollar en los estudiantes así como también estrategias de pensamiento, y en las unidades se detallan los contenidos conceptuales de cada una y algunas actividades experimentales sugeridas. Se constituyeron espontáneamente grupos de docentes que discutían propuestas didácticas, realizaban intercambios de bibliografía, materiales, etc. Se tomó en cuenta en forma creciente la teoría de Ausubel del aprendizaje significativo con énfasis en la naturaleza del aprendizaje y en las condiciones que se requieren para que el mismo pueda producirse en los alumnos.

Las reformas curriculares que comenzaron a aplicarse en algunos centros educativos en 1996 significaron cambios importantes en cuanto a la enseñanza de las ciencias experimentales. En Educación Secundaria se impartieron cursos de Ciencias de la Naturaleza (Biología, Física y Química) en primer y segundo año de $C B$ y de Ciencias Fisicoquímicas en el tercer año. Se realizaron acciones de capacitación para docentes, se editaron manuales orientadores del trabajo y libros de texto para los estudiantes.

Los documentos del Plan 1996 expresan los fundamentos de los cursos, plantean una forma enseñanza para lograr aprendizajes que sean funcionales, poniendo énfasis en laconsideración de las ideasprevias y los prerrequisitos. Se proponen contenidos de enseñanza, abarcando conceptos, estrategias, procedimientos y actitudes. Los programas contienen una selección de contenidos, sugerencias metodológicas y de actividades, indicadores de logros esperados en los estudiantes.

Esta reforma no contó con la adhesión de buena parte de los docentes por diversos motivos y fue revertida a partir del año 2006.

En el Consejo de Educación Técnico Profesional (CETP) se creó por entonces el Bachillerato en Química y también se reformularon varios programas. Resulta importante mencionar que los programas de los cursos del CETP, aún en el presente, se expresan en términos de competencias que se pretende desarrollar en los estudiantes, a través de los contenidos conceptuales que cada curso comporta. Ello implica considerar el aprendizaje explícitamente desde una perspectiva que fue muy cuestionada en el ámbito de la educación secundaria.
Posteriormente el CETP desarrolló la carrera de Tecnólogo Químico de nivel terciario, en convenio con la Universidad de la República. Los egresados de esta carrera adquieren una formación sobre los fundamentos de las técnicas de laboratorio usuales en control de industrial y sus aplicaciones.

Durante el año 2005 comenzaron a diseñarse nuevos programas en el CES, que aún se mantienen vigentes en la denominada Reformulación 2006. Los nuevos programas fueron elaborados de modo colaborativo, con participación de integrantes de la Asamblea Técnico Docente, profesores referentes de Química y de Didáctica de cada especialidad e inspectores. También fueron consultadas las salas docentes de todos los liceos del país. Estos nuevos programas de Química contienen en la parte inicial una fundamentación, objetivos generales de cada curso y algunas referencias generales acerca de los contenidos. Luego, por cada módulo temático, se plantean los objetivos generales y específicos que refieren a todos los dominios de conocimiento, las metas de aprendizaje, un listado de contenidos básicos (y en los programas de bachillerato además se incluyen contenidos de profundización y de contextualización), así como algunas sugerencias de actividades. Cierra cada módulo una profundización final con sugerencias metodológicas y de trabajo. Las propuestas se acompañan también de orientaciones para los aprendizajes en relación con el estudiante, el docente y el currículum. Se explicita también la concepción de evaluación de aprendizajes a utilizar y se presenta abundante bibliografía para el docente y el estudiante Se aprecia en estos programas una fuerte concepción del aprendizaje de enfoque constructivista, así como consideraciones acerca de las finalidades de la Educación Científica a través de la Química, tomando en cuenta tanto la necesidad de brindar a los educandos alfabetización científica y una formación para la vida así como también formación propedéutica, estableciéndose la conveniencia de un razonable equilibrio entre ambas metas.

\section{Formación Docente en nuestro país y Didáctica de la Química}

La Didáctica de Química -al igual que las Didácticas de las demás ciencias experimentales- también siguió las corrientes mundiales en general, pero las particularidades de la Formación Docente en el Uruguay ubican a nuestro país en una situación singular al respecto.

En los inicios de la Educación Secundaria los docentes de Química, al igual que los de las demás asignaturas, eranprofesionales destacados en la disciplina que ge- 
neralmente accedían a sus cargos mediante concursos de oposición y méritos, pero carecían de formación específica para la docencia.

La creación del Instituto de Profesores "Artigas" (IPA) tuvo lugar recién en 1950 comenzando al año siguiente las actividades sistemáticas, no alcanzando a cubrir el número de egresados las necesidades del sistema educativo en el nivel medio, especialmente en el área de las ciencias experimentales. Durante varias décadas para ser admitidos, los estudiantes eran seleccionados en base a los resultados de pruebas de ingreso de muy alta exigencia. La formación de los profesores se caracterizó desde sus orígenes hasta nuestros días por dedicar una parte al estudio de ciencias de la educación y otra a la formación en la disciplina y en didáctica, acompañando estos cursos con una práctica docente. Se buscaba formar un cuerpo de educadores de excelencia, estableciendo un perfil de docente de secundaria de alto nivel. Durante las primeras décadas de funcionamiento del IPA se desarrolló un programa de agregaturas con la finalidad de formar también a docentes para la enseñanza en este nivel, encontrándose escasas referencias en la especialidad Química.

Si bien la cantidad de docentes egresados fue creciendo con el tiempo, la extensión de la educación media llevó a no disponer de suficientes docentes formados para la función. Por ello muchos profesores accedían a la labor docente por concurso de oposición y méritos.

En 1976, se buscó homogeneizar la preparación de los docentes creando un "tronco común" a la formación de los educadores de Primaria, Secundaria y de Educación Técnica, con cursos de ciencias de la educación. Se creó una única institución en Montevideo para la formación de los docentes, el Instituto Nacional de Docencia (INADO), y en el interior se pasó la responsabilidad de la formación de docentes de educación media a los Institutos Normales. Una vez en democracia, se procedió a separar la formación de docentes para Enseñanza Secundaria de la de Primaria y se creó el Instituto Normal de Enseñanza Técnica (INET).

La formación de los profesores contaba con un curso de Didáctica General en el primer año con una práctica de observación en aula. En los dos años siguientes tenían lugar cursos de Didáctica específica con Práctica Docente. Durante cierto tiempo de la década del 70, la formación de los profesores de Química tuvo una carencia importante, ya que no se llevaban a cabo trabajos experimentales en los cursos específicos por limitaciones locativas y presupuestales. Con los cambios realizados en la formación docente a partir del año 1986, la misma se extendió a cuatro años, y se amplió la prepa- ración de docentes de Química a algunos Institutos de Formación Docente, como Paysandú en 1991 y Minas en 1992. En este plan los cursos de Didáctica pasaron a ser todos específicos, abandonando la perspectiva de una Didáctica General, con importantes referencias a las investigaciones didácticas que avanzaron en países desarrollados, desde una concepción constructivista del aprendizaje. A partir de entonces, son frecuentes las referencias en los programas a Ausubel, Vigotsky entre otros autores. Posteriormente se crearon los Centros Regionales de Profesores (CERP) facilitándose las oportunidades de formación de docentes al interior del país.Entre los años 1997 y 2000 se crean los CERP en Salto, Rivera, Maldonado, Colonia, Atlántida y Florida, sucesivamente. En su primera etapa el plan de estudios de estos centros expedía título de Profesor de Ciencias de la Naturaleza, con mención en Biología, Física o Química, según correspondiera. Podría considerarse que este plan tiene una concepción subyacente de Didáctica de las Ciencias, no tanto de didácticas específicas, aunque posteriormente, en el año 2008 se unificó la formación de profesores brindada en el IPA y en los CERP creándose el Sistema Único Nacional de Formación Docente (SUNFD). El Profesorado de Química, pasó a consistir en un tronco común de Ciencias de la Educación, asignaturas con contenidos disciplinares propios de la disciplina y Didáctica - Práctica Docente. La Práctica Docente se realiza durante tres años, en diferentes instituciones de educación media. Desde hace unos años este plan puede cursarse en modalidad presencial o semipresencial.

\section{La investigación en Didáctica de la Química}

Nuestro país no fue ajeno a los progresos de la Didáctica de la Química como disciplina científica en países desarrollados. Entre los años 1986 y 1988, un grupo de docentes de Biología y Química que trabajaban a nivel de formación docente como profesores del IPA o como docentes adscriptores de Educación Secundaria, comenzó a realizar reuniones intercambiando ideas sobre la didáctica y la investigación didáctica. El grupo tuvo la orientación de la Profesora de Química Beatríz Macedo -quien por entonces había obtenido el doctorado en Didáctica de la Química en París- y de la Profesora de Biología, residente en París, Mirtha Bazán Doctora en Didáctica de la Biología. Diversos intercambios francouruguayos, así como gestiones a nivel del Ministerio de Educación y Cultura hicieron posible que llegaran al Uruguay varios docentes franceses en visitas de cooperación, que contribuyeron a formar docentes en investigación didáctica. Recordamos en Química numerosas jornadas de trabajo con el Dr. George Soussan, el Dr. Maurice Chastrette, así como instancias de formación 
con docentes e investigadores de España como el Dr. Carlos Furió Mas. Paralelamente, el grupo de trabajo presentó a las autoridades de la educación la propuesta de creación del Laboratorio de Investigación Didáctica (LID) en el ámbito del Instituto de Profesores "Artigas", el cual funcionó como espacio de investigación desde 1988 hasta febrero de 1997. Una vez creado el LID se llevó a cabo una importante investigación a nivel nacional indagando en profundidad acerca de las concepciones de los docentes de Biología y Química en el plano didáctico, la cual fue divulgada en 1992. Posteriormente, las profesoras de Química integrantes de este laboratorio llevaron adelante otra investigación sobre las relaciones entre la apropiación de conceptos y la resolución de problemas en Química entre 1994 y 1996. Por otra parte, se publicaron varios boletines para la difusión entre los docentes; entre ellos sedestaca uno dedicado a las actividades prácticas. La producción del LID contribuyó a que muchos docentes y autoridades de diversos organismos pensaran en la necesidad de promover la investigación educativa y llevar a cabo investigación didáctica en nuestro país. Por ejemplo, la Facultad de Química de la UdelaR, así como algunas universidades privadas comenzaron a ofrecer estudios de posgrado que incluyen actividades de investigación, muchas veces de corte pedagógico más que didáctico, pero que contribuyen a la producción de conocimiento acerca del ámbito educativo nacional.

Una vez quese comenzó la adjudicación de horas de Coordinación y de Departamento a los docentes del CFE, muchos profesores comenzaron a realizar variadas investigaciones, algunas de las cuales refieren a la didáctica de la química.

La puesta en marcha de los Posgrados en Didáctica a nivel medio entre los que se ofreciólaorientación en Didáctica de la Química, en el Instituto de Perfeccionamiento y Estudios Superiores (IPES) en 2008 permitió la producción de varios proyectos de investigación en este campo y de Tesinas del diplomado. Lamentablemente, no se llegó a concretar el desarrollo de las Maestrías en Didáctica que habían sido aprobadas inicialmente.Por otra parte, en el marco de los estudios de posgrado, la Facultad de Química de la UdelaR ofrece desde mediados de la década del 2000 la posibilidad de obtener una Maestría en Química con mención en Educación. Como parte de esos estudios se realizan actividades de investigación, algunas de las cuales constituyen investigaciones didácticas. Con todos estos insumos, se está comenzando a disponer de un cuerpo de conocimientos didácticos propios de nuestro país de importante valor.

\section{Otros aportes a la formación de profesores de Química.}

La apertura democrática posibilitó por un lado el inicio de los Congresos Nacionales e Internacionales de profesores de Química, el primero de ellos en la ciudad de Artigas en 1987 y la fundación de la Asociación de Educadores en Química (ADEQ) en el año 1988. Esta asociación se inició mayormente con profesores de Educación Secundaria, pero pronto contó con profesores de otros subsistemas, así como con socios docentes de Facultad de Química y algunos profesores extranjeros. Entre sus principales objetivos se encuentra contribuir a la formación permanente de los profesores de esta disciplina. Realiza actividades variadas como conferencias ycursillos; desde hace algunos años ha tomado a su cargo la organización de los congresos Nacionales e Internacionales de Profesores de Química que ya son tradicionales en nuestro medio.

\section{Reflexiones finales}

La enseñanza de la química en nuestro país ha mostrado una interesante evolución desde sus inicios hasta el presente. También, en consonancia con lo ocurrido en otros países, su didáctica ha experimentado una evolución transformándose en una disciplina científica con un campo de conocimientos propio, nutriéndose de los aportes de la psicología, la epistemología y la sociología entre otras disciplinas. Los cambios de paradigma en las concepciones de aprendizaje, del trabajo científico, de la ciencia como parte de la cultura, se aprecian en este recorrido. De todas maneras, los permanentes cambios que se dan en la sociedad, en todas sus dimensiones, nos obliga a pensar permanentemente las prácticas educativas en esta disciplina en constante crecimiento. Siempre habrá que seleccionar los contenidos que resulten más relevantes así como las estrategias didácticas que permitan a nuestros estudiantes construir saberes disciplinares, apropiarse del conocimiento químico y crecer como personas, mientras disfrutan de la belleza de este conocimiento, como remarcaba Marie Curie.

\section{Referencias bibliográficas}

Alonzo, D. (2010). Síntesis histórica de UTU. Recuperado http://profdayanaalonzo.blogspot.com/2010/03/utusintesis-historica.htm

Programa de Educación Inicial y Primaria. Año 2008. CEIP. Recuperado (5/10/2010).

Verdesio, E. (1962). Génesis de laeducación uruguaya. Imprenta Nacional. Montevideo. 